Artigo Original

\title{
Comparação do Ponto de deflexão da frequência cardíaca com a Máxima fase estável de lactato em corredores de fundo
}

\author{
Bruno Honorato da Silveira \\ Rafael Alves de Aguiar \\ Thiago Luiz Alves \\ Fabrizio Caputo \\ Lorival José Carminatti \\ Laboratório de Pesquisas em Desempenho Humano, Centro de Ciências da Saúde e do
Esporte, Universidade do Estado de Santa Catarina (UDESC), Florianópolis, SC, Brasil \\ Resumo: O objetivo do estudo foi comparar o ponto de deflexão da freqüência cardíaca (PDFC) visual e \\ método DMAX com a máxima fase estável de lactato (MFEL). Treze corredores executaram teste \\ incremental Vameval e testes de cargas retangulares (TCR). A velocidade do PDFC visual (14,3 \pm \\ $\left.1,13 \mathrm{~km} \cdot \mathrm{h}^{-1}\right)$ foi significantemente maior que o $\operatorname{DMAX}\left(13,2 \pm 1,35 \mathrm{~km} \cdot \mathrm{h}^{-1}\right)$ além de apresentarem correlação \\ não significante. Entretanto, nenhuma dessas velocidades foram diferentes da MFEL $\left(13,8 \pm 0,90 \mathrm{~km} . \mathrm{h}^{-1}\right)$ \\ embora somente o PDFC visual tenha apresentado correlação significante com a MFEL $(r=0,75)$. A \\ concentração de lactato sanguíneo não apresentou estabilidade em oito sujeitos no TCR na intensidade do \\ PDFC visual o qual nos leva a concluir que este não é um índice confiável para estimativa da MFEL. No \\ entanto, este índice pode ser usado como um indicador de capacidade aeróbia.
}

Palavras-chave: Ponto de deflexão da freqüência cardíaca. Máxima fase estável de lactato. Corrida de fundo.

\section{Comparison between Heart rate deflection point and Maximal lactate steady state in distance runners}

\begin{abstract}
The aim of study was to compare heart rate deflection point (HRDP) determined by visual and DMAX methods to Maximal lactate steady state (MLSS). Thirteen runners carried out incremental test Vameval and constant load tests (CLT). Velocity of HRDP $\left(14,3 \pm 1,13 \mathrm{~km} \cdot \mathrm{h}^{-1}\right)$ was significantly higher compared to DMAX $\left(13,2 \pm 1,35 \mathrm{~km} . \mathrm{h}^{-1}\right)$ but they were not significantly correlated. However, both velocities, HRDP and DMAX, were not different from MLSS $\left(13,8 \pm 0,90 \mathrm{~km} . \mathrm{h}^{-1}\right)$ while only HRDP has been significant correlated with MLSS $(r=0,75)$. On eight runners during CLT the blood lactate concentration did not show stability at HRDP velocity which to let us to conclude that HRPD is not appropriated to estimate MLSS. However, it may be used as aerobic capacity index
\end{abstract}

Key-words: Heart rate deflection point. Maximal lactate steady state. Endurance runners.

\section{Introdução}

Muitas são as variáveis fisiológicas que interferem na performance em corrida de fundo, nas quais se destacam a velocidade máxima aeróbia, a máxima fase estável de lactato (MFEL) e a economia de movimento (MORGAN et al, 1989; BILLAT, 1996; DENADAI; ORTIZ; MELLO, 2004). A MFEL, particularmente, vem recebendo uma grande atenção por pesquisadores do esporte uma vez que se apresenta como um importante índice fisiológico para a predição da performance, em provas em que a produção de energia ocorra predominantemente a partir do sistema aeróbio (DENADAI, 1999). Como conceito, a MFEL representa a máxima intensidade de trabalho que pode ser sustentada sem uma contínua acumulação de lactato sanguíneo durante exercício de intensidade constante (BENEKE, 2003). Entretanto, sua determinação requer sucessivas visitas com aplicação de testes com carga retangular (TCR), em diferentes dias, com aproximadamente $30 \mathrm{~min}$ de duração (MADER, 1991; BENEKE, 2003).

$\mathrm{Na}$ tentativa de reduzir a quantidade de testes, uma estimativa da MFEL foi proposta através da utilização de protocolos de teste incremental (HECK et al, 1985). Heck et al (1985) aplicaram teste incremental com estágios de três e cinco minutos na corrida e denominaram limiar anaeróbio, determinado através de concentrações fixas de lactato, como sendo a intensidade estimada da MFEL. O limiar anaeróbio parece ter associação com a MFEL determinada de forma convencional, sendo eficaz no intuito de diminuir a 
quantidade de visitas ao laboratório. Entretanto, ainda é inviável para a maioria dos treinadores e corredores por se tratar de um procedimento invasivo e de alto custo financeiro (BODNER; RHODES, 2000; PASSELERGUE et al, 2006; HOFMANN et al., 2007).

O ponto de deflexão da freqüência cardíaca (PDFC) visual proposto por Conconi et al. (1982) assume o pressuposto de que a freqüência cardíaca aumenta de maneira linear, juntamente com a carga de trabalho, até o momento em que tende a estabilizar, independente dos incrementos da intensidade. Dessa forma, o PDFC visual tem sido bastante utilizado para a identificação do

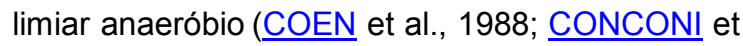
al., 1996), trazendo como vantagens a simplicidade e a característica de não ser um método invasivo necessitando apenas do monitoramento da frequencia cardiaca (FC). Outros estudos foram também realizados com 0 objetivo de descrever as respostas fisiológicas durante exercícios de carga constante na intensidade referente ao PDFC visual (BOURGOIS; VRIJENS, 1998; BOURGOIS et al., 2004). Remadores (BOURGOIS; VRIJENS, 1998) e Ciclistas (BOURGOIS et al., 2004) realizaram uma única sessão de exercício constante na intensidade do PDFC visual. Nesses dois estudos, a maioria dos sujeitos não terminou a duração proposta, além de não apresentarem estabilidade na concentração de lactato sanguíneo. Resultados semelhantes foram também encontrados em corredores que realizaram um exercício constante levemente abaixo $\left(\sim 0,5 \mathrm{~km} . \mathrm{h}^{-}\right.$ $\left.{ }^{1}\right)$ da velocidade do PDFC visual (JONES; DOUST, 1997). Esses resultados sugerem que a intensidade do PDFC visual pode não representar uma estabilidade fisiológica, tendo como parâmetro a concentração de lactato sanguíneo.

Além dos aspectos relacionados às respostas fisiológicas na intensidade do PDFC visual em alguns trabalhos não foi possível encontrar o PDFC visual em todos os sujeitos (JONES; DOUST, 1995; 1997; VACHON; BASSETT; CLARKE, 1999; PASSELERGUE et al., 2006; COSTA et al., 2007). Posteriormente ao PDFC visual surgiu um método mais objetivo que o empregado por Conconi e colaboradores (1982) com ajuste matemático da resposta da FC no teste incremental. $\mathrm{O}$ método emprega um ajuste polinomial de terceira ordem e determinação de uma reta, utilizando dois pontos para a sua determinação: o primeiro valor superior ou igual a
140 bpm e o segundo a freqüência cardíaca máxima (FCmáx), assim o PDFC pelo ajuste matemático foi considerado como o ponto mais distante entre a reta e a curva ajustada (Dmax) (KARA et al., 1996). Segundo os autores, a utilização deste método além de diminuir a subjetividade implícita na determinação visual do PDFC, possibilita a identificação positiva do fenômeno na totalidade dos casos. Assim, a hipótese do presente estudo é que o DMAX reduz a subjetividade do método visual aproximando o fenômeno do PDFC à MFEL. Nesse sentido, o objetivo do estudo foi comparar o PDFC visual e DMAX com a MFEL em corredores.

\section{Metodologia}

\section{Amostra}

Participaram desse estudo treze corredores de provas de fundo (30 $\pm 8,48$ anos; $171,3 \pm 7,14 \mathrm{~cm}$; $70,4 \pm 8 \mathrm{~kg} ; 7,2 \pm 4,40 \%$ gordura). Como critério de inclusão foram considerados a idade (20-45 anos), período mínimo de dois anos de treinamento regular e performance nos $10 \mathrm{~km}$ entre 35 e 50min. O estudo foi aprovado pelo Comitê de Ética da Instituição no qual foi realizado o estudo (Protocolo $n^{\circ} 47 / 2009$ ). Todos os atletas foram informados sobre os objetivos, procedimentos da pesquisa e dos possíveis riscos envolvidos no estudo e logo após assinaram o termo de consentimento livre e esclarecido, concordando em participar voluntariamente da pesquisa.

\section{Procedimentos}

Todas as avaliações do estudo foram realizadas na pista de atletismo sintética e descoberta com perímetro $200 \mathrm{~m}$. Os avaliados foram orientados a não realizar nenhum tipo de exercício físico intenso no período de 24 horas que antecederam cada sessão de teste.

As avaliações tiveram início a partir da avaliação antropométrica: a massa corporal (MC) foi mensurada com uma balança digital de precisão $100 \mathrm{~g}$ marca Toledo $\AA$; a estatura (ES) foi obtida em um estadiômetro profissional de precisão $0,5 \mathrm{~cm}$ da marca Cescorf®; o percentual de gordura foi determinado a partir da equação de três dobras cutâneas (peitoral, abdômen e coxa média) proposta por Jackson e Pollock (1978) com uso do adipômetro científico da marca Cescorf®. A temperatura e umidade relativa do ar foram registradas durante os testes com um termômetro/higrômetro digital da marca 
Vacumed $®$. Todos os testes foram realizados em uma pista de atletismo descoberta com valores 23 $\pm 3,76^{\circ} \mathrm{C}$ e $56 \pm 11,65 \%$ de temperatura e umidade relativa do ar, respectivamente.

\section{Teste Incremental de corrida Vameval (TVAM)}

Para o teste incremental de corrida foi adotado o TVAM muito utilizado em centros de rendimento (CAZORLA, 1990), com velocidade inicial de $8,5 \mathrm{~km} \cdot \mathrm{h}^{-1}$ e incrementos de $0,5 \mathrm{~km} \cdot \mathrm{h}^{-1}$ a cada estágio de duração $\sim 1 \mathrm{~min}$ ). $\mathrm{O}$ ritmo de corrida foi controlado por meio da emissão de sinais sonoros (bips) e cones de "pvc" distribuídos na pista a cada $20 \mathrm{~m}$.

Os sujeitos foram orientados a realizar um teste máximo, recebendo incentivo verbal durante os estágios finais do TVAM. O teste foi encerrado por exaustão voluntária ou quando o atleta não conseguiu manter o ritmo imposto pelo protocolo (atraso a uma distância superior a $2 \mathrm{~m}$ em três cones consecutivos). A velocidade final do TVAM foi denominada Pico de velocidade (PV) e nos casos em que o sujeito interrompeu o teste antes de finalizar um estágio do protocolo, a velocidade final foi corrigida a partir da seguinte equação:

$$
\mathrm{PV}\left(\mathrm{km} \cdot \mathrm{h}^{-1}\right)=\mathrm{v}+[(\mathrm{nv} / \mathrm{tv}) \cdot 0,5]
$$

Onde: "v" é a velocidade do último estágio completo em $\mathrm{km} \cdot \mathrm{h}^{-1}$, o "nv" é o número de voltas percorridas no estágio incompleto, "tv" é o número total de voltas do estágio não completado (adaptado de Kuipers et al. (1985)).

A FCmax foi considerada o maior valor registrado no final do teste concomitantemente com o $\mathrm{PV}$, sendo considerado como critério de teste máximo a FC ter atingido pelo menos $90 \%$ da $\mathrm{FCmax}$ predita pela idade $\left(\mathrm{FCmax}_{\text {pred }}=220-\right.$ idade).

A FC foi registrada por meio de um monitor de FC da marca Polar Electroß - modelo S610, armazenadas em intervalos de 5 segundos para obtenção do PDFC visual e o DMAX. A transição de um aumento linear (ou quase linear) da $\mathrm{FC}$ em cargas intermediárias para um incremento não linear em cargas mais altas foi definida como a intensidade do PDFC. Para a análise visual do PDFC, individualmente três avaliadores experientes determinaram o PDFC visual identificando a respectiva velocidade e FC. A partir da identificação dos três avaliadores foi adotada a moda para determinação do PDFC visual; em todas as análises do PDFC visual foi possível encontrar a moda. No método matemático DMAX os pontos foram ajustados por uma função polinomial de terceira ordem e, posteriormente foi empregado um ajuste linear, utilizando-se os dois extremos da curva, derivando-se com uma reta. O DMAX foi definido como o ponto mais distante entre as duas linhas (KARA et al., 1996), sendo utilizado apenas valores de $\mathrm{FC} \geq 140 \mathrm{bpm}$ (LEITNER; HOFMANN; GAISL, 1988; KARA et al., 1996).

\section{Teste de Carga Retangular (TCR)}

Foi adotado antes de todas as sessões de carga retangular um aquecimento de $5 \mathrm{~min}$ à velocidade de $60 \% \mathrm{PV}$ com intervalo passivo de 3 min para o início da primeira série do TCR. Cada sessão de TCR teve duração de $30 \mathrm{~min}$ divididos em três séries de $10 \mathrm{~min}$ de corrida e intervalo de $1 \mathrm{~min}$. Logo após o aquecimento e nos intervalos das séries do TCR foram coletadas amostras de $25 \mu \mathrm{L}$ de sangue capilar do lóbulo da orelha (previamente hiperemiada com pomada Finalgon () e a análise da concentração de lactato sanguíneo foi realizada imediatamente no aparelho YSI 1500 Sport巴 (EUA); ainda nos intervalos foi mensurado a FC.

O primeiro TCR foi realizado com a velocidade correspondente ao PDFC visual. Subseqüentes TCRs foram aplicados nos intervalos de 2 a 5 dias com variação de 2 a $5 \%$ do PV (para mais ou para menos) sobre a velocidade do PDFC visual até que a MFEL fosse confirmada. A MFEL foi definida como a maior velocidade em que ocorreu a fase estável de lactato, aumento não superior a $1,0 \mathrm{mmol} \cdot \mathrm{I}^{-1}$ entre o $10^{\circ}$ e o $30^{\circ}$ minuto (Beneke, 2003).

\section{Análise estatística}

Foi aplicado o teste de normalidade ShapiroWilk para verificar a normalidade dos dados. Os dados foram tratados pela estatística paramétrica e apresentados pela estatística descritiva (média, desvio-padrão, mínimo e máximo, coeficiente de variação). A comparação entre as velocidades da MFEL, PDFC visual e DMAX foi realizada pela Anova Oneway e, quando foi encontrada diferença, utilizou-se o post-hoc de Tukey. A correlação entre as variáveis foi verificada pelo coeficiente de correlação de Pearson. Em todos os testes foi adotado o nível de significância de $5 \%$ (programa SPSS $®$ v. 17.0) 


\section{Resultados}

Nenhuma das velocidades referentes ao PDFC visual e DMAX se diferenciou da MFEL (tabela 1). Contudo, a velocidade do PDFC visual foi significantemente maior do que a do DMAX. Ambas as velocidades da MFEL e PDFC visual tiveram correlação moderada com o PV $(r=0,66 ; 0,68$, respectivamente). Com uma correlação forte, o PDFC visual $(r=0,75)$ foi o único entre os métodos de identificação do PDFC que tiveram associação com a MFEL (tabela 2).

Tabela 1- Resultados médios (média, desvio padrão e coeficiente de variação) das variáveis fisiológicas.

\begin{tabular}{cccccc}
\hline & PV & FCmax & VMFEL & VPDFC visual & VDMAX \\
\hline Média & 17,1 & 183 & 13,8 & $14,3^{*}$ & 13,2 \\
DP & 0,95 & 10,04 & 0,90 & 1,13 & 1,35 \\
CV & 5,55 & 5,49 & 6,57 & 7,88 & 10,22 \\
\hline
\end{tabular}

VMFEL = velocidade da máxima fase estável de lactato; VPDFC = velocidade do método visual do ponto de deflexão da freqüência cardíaca; VDMAX= velocidade do método matemático do ponto de deflexão da freqüência cardíaca.

"Diferença significante em relação ao DMAX $(p<0,05)$.

Tabela 2- Correlações entre VMFEL, VPDFC visual, VDMAX e PV.

\begin{tabular}{cccc}
\hline & VMFEL & VPDFC visual & VDMAX \\
\hline VMFEL & ------ & $0,75^{* *}$ & 0,42 \\
VPDFC visual & $0,75^{\star *}$ & --- & 0,08 \\
VDMAX & 0,42 & 0,08 & ---- \\
PV & $0,66^{*}$ & $0,68^{* *}$ & 0,28 \\
\hline
\end{tabular}

${ }^{*}$ Correlação significante $(p<0,05)$.

** Correlação significante $(p<0,01)$.

Pode ser observado na tabela 3, o comportamento da concentração do lactato sanguíneo e FC na velocidade do PDFC visual durante o TCR. O coeficiente de variação do erro típico da estimativa da velocidade da MFEL em relação ao PDFC visual e DMAX foram 4,7 e 6,5\%, respectivamente.

Tabela 3- Resposta individual da concentração de lactato sanguíneo (mmol..- $\left.{ }^{1}\right)$ e FC (bpm) na velocidade do PDFC visual no TCR.

\begin{tabular}{ccccccc}
\hline Sujeitos & $\begin{array}{c}\text { Tempo } \\
(\mathrm{min})\end{array}$ & $\begin{array}{c}\text { Lac } \\
10 \mathrm{~min}\end{array}$ & Lac Final & $\Delta$ Lac & $\begin{array}{c}\text { FC 10 } \\
\text { min }\end{array}$ & $\begin{array}{c}\text { FC } \\
\text { Final }\end{array}$ \\
\hline 1 & 10 & 17,09 & 18,41 & 1,32 & 170 & 170 \\
2 & 30 & 6,37 & 7,75 & 1,38 & 181 & 191 \\
3 & 30 & 3,96 & 4,63 & 0,67 & 166 & 168 \\
4 & 30 & 2,69 & 3,02 & 0,33 & 171 & 174 \\
5 & 30 & 8,12 & 9,63 & 1,51 & 154 & 180 \\
6 & 17,7 & 9,98 & 11,64 & 1,66 & 185 & 191 \\
7 & 30 & 5,01 & 5,29 & 0,28 & 152 & 159 \\
8 & 30 & 5,26 & 8,59 & 3,33 & 165 & 168 \\
9 & 30 & 5,01 & 6,06 & 1,05 & 175 & 179 \\
10 & 30 & 5,43 & 9,53 & 4,01 & 189 & 197 \\
11 & 30 & 2,51 & 2,67 & 0,16 & 165 & 160 \\
12 & 22,7 & 5,72 & 7,38 & 1,66 & 169 & 175 \\
13 & 30 & 7,13 & 8,03 & 0,9 & 174 & 176 \\
\hline Média & 26,95 & 6,48 & 7,89 & 1,40 & 170 & 176 \\
DP & 6,35 & 3,78 & 4,11 & 1,14 & 10,78 & 12,03 \\
\hline
\end{tabular}




\section{Discussão}

O objetivo do nosso estudo foi comparar o PDFC visual e DMAX com a MFEL em corredores de fundo. A hipótese de que o DMAX reduz a subjetividade do método visual aproximando o fenômeno do PDFC à MFEL foi rejeitada. O principal achado do nosso estudo foi que apenas - PDFC visual apresentou validade moderada como estimativa da intensidade de MFEL, sugerindo que este índice pode ser usado como um indicador de capacidade aeróbia.

No presente estudo foi possível identificar o PDFC em todos os sujeitos pela inspeção visual (CONCONI et al., 1982) e por meio do método matemático DMAX (KARA et al., 1996). O DMAX tem como pressuposto ser um método mais objetivo para identificação do PDFC (KARA et al., 1996) do que a inspeção visual. Kara et al. (1996) também identificaram o PDFC em todos os sujeitos $(n=32)$ por meio do método DMAX, entretanto, em apenas 23 sujeitos foi possível a identificação pelo método visual do PDFC, diferentemente do nosso estudo que houve identificação positiva em $100 \%$ dos sujeitos em ambos os métodos. A subjetividade tem sido apontada por alguns estudos como uma desvantagem do método de Conconi dificultando a identificação do fenômeno (JONES; DOUST, 1995; BODNER; RHODES, 2000). Dificuldade esta que foi amenizada no corrente trabalho pelo uso da medida moda de três diferentes avaliadores experientes nas respectivas velocidade e freqüência cardíaca do PDFC visual.

Outro aspecto a ser considerado na determinação do PDFC visual é o tipo de protocolo utilizado. No protocolo do teste incremental utilizado em nosso estudo (Vameval), o aumento da velocidade se deu com pequenos incrementos em estágios de tempo fixo, o que vai de acordo com as recomendações de Conconi et al (1996). Entretanto, no trabalho de Kara et al. (1996) não foi descrito de maneira clara o protocolo incremental realizado. Assim, não se pode estabelecer precisamente se o protocolo adotado (KARA et al., 1996) foi adequado para identificação do fenômeno do PDFC visual.

É conhecido na literatura que a curva da FC pode apresentar tanto uma curvatura côncava como convexa (BODNER; RHODES, 2000;
COSTA et al., 2007). O método DMAX aplica um ajuste matemático que identifica a maior distância entre a curva de FC e a reta unindo os dois pontos extremos independente da característica da curva de FC. Além disso, pequenas variações na curva de FC também poderiam interferir na precisão do fenômeno determinado pelo DMAX. Assim, podemos especular que o êxito na identificação do PDFC em todos os sujeitos no método DMAX pode ser devido à manipulação matemática. Contudo, a abordagem matemática foge do escopo do nosso trabalho.

Ainda em relação aos achados de Kara et al. (1996), os autores não observaram diferenças entre as intensidades do PDFC visual e DMAX, além de uma forte correlação entre os métodos. Esses resultados contradizem aos nossos achados, no qual o PDFC visual e DMAX foram diferentes e não correlacionados. Além disso, apesar do nosso estudo não ter encontrado diferença entre a velocidade de MFEL e DMAX, a baixa correlação entre essas duas variáveis sugere que as mesmas não estariam representando o mesmo fenômeno fisiológico. Uma possível explicação para esses resultados pode ser apontada pelas características descritas acima em relação ao DMAX e pela realização do teste incremental em campo. Em nosso trabalho, a velocidade dos sujeitos foi controlada pela emissão de bips o que pode ter influenciado o ajuste cardiovascular durante o teste incremental. No entanto, estes aspectos foram minimizados pela utilização de marcações a cada $20 \mathrm{~m}$. Nesse sentido uma explicação definitiva para diferença encontrada entre PDFC visual e DMAX não pode ser providenciada e qualquer outra sugestão continuaria a ser meramente especulativa.

Conconi et al (1982) propuseram inicialmente um método não-invasivo que seria coincidente ao limiar anaeróbio e de fácil aplicação em teste de campo. Baseado na validade do limiar anaeróbio com a MFEL (HECK et al., 1985; SIMOES et al., 1999), alguns trabalhos utilizaram o limiar anaeróbio como estimativa da MFEL na comparação com o PDFC visual (HOFMANN; LEITNER; GAISL, 1992; HOFMANN et al., 1994; BUNC et al., 1995; BOURGOIS et al., 2004). Em nosso estudo não foi observado diferença entre as velocidades do PDFC visual e MFEL, além de uma correlação significante $(r=0,75)$. Corroborando com os nossos resultados, alguns estudos verificaram valores semelhantes em variáveis metabólicas e cardiovasculares 
relacionados ao PDFC visual e limiar anaeróbio em ciclo ergômetro (HOFMANN; LEITNER; GAISL, 1992; HOFMANN et al., 1994; BUNC et al., 1995; BOURGOIS et al., 2004). Portanto, esses achados juntamente com os nossos resultados sugerem o PDFC visual ser um indicador de capacidade aeróbia.

$\mathrm{Na}$ tentativa de analisar o comportamento do estado metabólico, os sujeitos do nosso estudo realizaram exercício constante na velocidade do PDFC visual durante $30 \mathrm{~min}$. Observamos dois padrões de comportamento do lactato sanguíneo nessa intensidade entre os treze corredores. Primeiro, em cinco sujeitos a concentração de lactato sanguíneo apresentou estabilidade. Segundo, outros oito indivíduos não alcançaram estabilidade no lactato sanguíneo, sendo que três sequer terminaram os $30 \mathrm{~min}$ propostos. Para o grupo como um todo, a duração média do TCR foi $26,95 \pm 6,35 \mathrm{~min}$. Respostas metabólicas similares em exercício na intensidade do PDFC visual foram observadas por Bourgois e Vrijens (1998) e Bourgois et al. (2004) onde somente um $(n=10)$ e quatro ( $n=11$ ) sujeitos demonstraram equilíbrio na concentração de lactato sanguíneo, respectivamente. Além disso, o tempo médio dos estudos acima foi similar ao nosso (17,5 \pm $11,1 \mathrm{~min}$ e $23,8 \pm 8,8 \mathrm{~min}$ respectivamente). Ainda em intensidade ligeiramente inferior ao PDFC visual $(-2,8 \%)$, nenhum corredor $(n=7)$ demonstrou equilíbrio nas concentrações de lactato (JONES; DOUST, 1997). Dessa forma, parece que o PDFC visual pode não ser um real marcador do limite superior do domínio pesado devido à ausência da estabilidade nas concentrações de lactato sanguíneo para a maioria dos sujeitos.

$\mathrm{Na}$ tentativa de aumentar a precisão da identificação da MFEL optamos por uma aplicação com pequenas variações de intensidade $(3,4 \%$, em média) entre os TCRs. Apesar de não ter sido encontrada diferença estatística entre as intensidades do PDFC visual e MFEL, os valores de erro típico $(4,7 \%)$ foram maiores que aqueles adotados entre os TCRs para determinação da MFEL demonstrando que esta magnitude de erro na estimativa da intensidade da MFEL, apesar de não ser elevada, é suficiente para indicar ausência de estabilidade no lactato sanguíneo. Característica que foi parcialmente confirmada pelas respostas metabólicas do TCR na intensidade do PDFC visual. A ausência da diferença entre as intensidades do PDFC visual e
MFEL encontrada no nosso estudo pode ser explicado, pelo menos em parte, pelo modelo experimental (múltiplas comparações e número de sujeitos testados) o qual pode ter influenciado o significado estatístico dos resultados, acarretando um erro do tipo 2 na análise estatística.

Em conclusão, o nosso estudo demonstrou que o PDFC visual é um bom indicador de capacidade aeróbia. Entretanto, o PDFC visual deve ser utilizado com cautela como estimativa indireta da intensidade de MFEL. Assim, para situações nas quais uma maior precisão é importante seria necessário a determinação direta da MFEL. Pelo menos nas condições experimentais do nosso estudo, o DMAX não está associado aos mesmos fenômenos fisiológicos apresentados pelo PDFC visual e MFEL.

\section{Referências}

BENEKE, R. Methodological aspects of maximal lactate steady state-implications for performance testing. European Journal of Applied

Physiology, Basel, v. 89, n. 1, p. 95-9, 2003.

BILLAT, L. V. Use of blood lactate measurements for prediction of exercise performance and for control of training: recommendations for longdistance running. Sports Medicine, Auckland, v. 22, n. 3, p. 157-75, 1996.

BODNER, M. E.; RHODES, E. C. A review of the concept of the heart rate deflection point. Sports Medicine, Auckland, v. 30, n. 1, p. 31-46, 2000.

BOURGOIS, J.; VRIJENS, J. The conconi test: a controversial concept for the determination of the anaerobic threshold in young rowers. International Journal of Sports Medicine, Stuttgart, v. 19, n. 8, p. 553-9, 1998.

BOURGOIS, J.; COOREVITS, P.; DANNEELS, L.; WITVROUW, E.; CAMBIER, D.; VRIJENS, J. Validity of the heart rate deflection point as a predictor of lactate threshold concepts during cycling. Journal of Strength and Conditioning Research, Philadelphia, v. 18, n. 3, p. 498-503, 2004.

BUNC, V.; HOFMANN, P.; LEITNER, H.; GAISL, $G$. Verification of the heart rate threshold.

European Journal of Applied Physiology and Occupational Physiology, Basel, v. 70, n. 3, p. 263-269, 1995.

CAZORLA, G. Test de terrain pour evaluer la capacite aerobie et la vitesse aerobic maximale. In: ACTES DU COLLOQUE INTERNATIONAL DE 
LA GUADELOUPE, 1990. Guadeloupe. Actes... Guadeloupe: [s.n.], 1990. p.151-173.

COEN, B.; URHAUSEN, A.; KINDERMANN, W. Value of the Conconi test for determination of the anaerobic threshold. Journal of Sports

Sciences, London, v. 9, p. 372, 1988.

CONCONI, F.; FERRARI, M.; ZIGLIO, P. G.; DROGHETTI, P.; CODECA, L. Determination of the anaerobic threshold by a noninvasive field test in runners. Journal of Applied Physiology, Bethesda, v. 52, n. 4, p. 869-873, 1982.

CONCONI, F.; GRAZZI, G.; CASONI, I.; GUGLIELMINI, C.; BORSETTO, C.; BALLARIN, E.; MAZZONI, G.; PATRACCHINI, M.; MANFREDINI, F. The Conconi test: methodology after 12 years of application. International Journal of Sports Medicine, Stuttgart, v. 17, n. 7, p. 509-519, 1996.

COSTA, V. P. da; KARASIAK, F. C.; FRONCHETTI, L.; KROEFF, M. S. Identificação do ponto de deflexão da frequência cardíaca. Revista Treinamento Desportivo, Curitiba, v. 8, p. 71-76, 2007.

DENADAI, B. S. Índices fisiológicos de avaliação aeróbia: conceitos e aplicações. Ribeirão Preto: BSD, 1999.

DENADAI, B. S.; ORTIZ, M. J.; MELLO, M. T. de. Índices fisiológicos associados com a "performance" aeróbia em corredores de "endurance": efeitos da duração da prova. Revista Brasileira de Medicina do Esporte, São Pualo, v. 10, p. 401-404, 2004.

HECK, H.; MADER, A.; HESS, G.; MUCKE, S.; MULLER, R.; HOLLMANN, W. Justification of the 4-mmol/l lactate threshold. International Journal of Sports Medicine, Stuttgart, v. 6, n. 3, p. 117130, 1985.

HOFMANN, P.; LEITNER, H.; GAISL, G. Heart rate threshold, lactate turn point and anaerobic threshold determination by electromyography. Hungarian Review of Sports Medicine, Warsaw, v. 33, p. 13-20, 1992.

HOFMANN, P.; BUNC, V.; LEITNER, H.; POKAN, R.; GAISL, G. Heart rate threshold related to lactate turn point and steady-state exercise on a cycle ergometer. European Journal of Applied Physiology and Occupational Physiology, Berlin, v. 69, n. 2, p. 132-139, 1994.

HOFMANN, P.; JURIMAE, T.; JURIMAE, J.; PURGE, P.; MAESTU, J.; WONISCH, M.; POKAN, R.; VON DUVILLARD, S. P. HRTP, prolonged ergometer exercise, and single sculling.
International Journal of Sports Medicine, Stuttgart, v. 28, n. 11, p. 964-969, 2007.

JACKSON, A. S.; POLLOCK, M. L. Generalized equations for predicting body density of men. The British Journal of Nutrition, Cambridge, v. 40, n. 3, p. 497-504, 1978.

JONES, A. M.; DOUST, J. H. Lack of reliability in Conconi's heart rate deflection point.

International Journal of Sports Medicine, Stuttgart, v. 16, n. 8, p. 541-544, 1995.

JONES, A. M.; DOUST, J. The Conconi test is not valid for estimation of lactate tumpoint in runners. Journal of Sports Sciences, Oxon, v. 15, p. 385394, 1997.

KARA, M.; GOKBEL, H.; BEDIZ, C.; ERGENE, N.; UCOK, K.; UYSAL, H. Determination of the heart rate deflection point by the Dmax method. The Journal of Sports Medicine and Physical Fitness, Torino, v. 36, n. 1, p. 31-34, 1996.

KUIPERS, H.; VERSTAPPEN, F. T.; KEIZER, H. A.; GEURTEN, P.; VAN KRANENBURG, G. Variability of aerobic performance in the laboratory and its physiologic correlates. International Journal of Sports Medicine, Stuttgart, v. 6, n. 4, p. 197-201, 1985.

LEITNER, H.; HOFMANN, P.; GAISL, G. A method for the microcomputeraided determination of the anaerobic threshold by means of heart rate curve analysis. In: CONFERENCE PROCEEDINGS ON BIOMEDICAL ENGINEERING IN AUSTRIA, 1987, Austria. Proceedings... Austria: [s.n.], 1987. p.136-141.

MADER, A. Evaluation of the endurance performance of marathon runners and theoretical analysis of test results. The Journal of Sports Medicine and Physical Fitness, Torino, v. 31, n. 1, p. 1-19, 1991.

MORGAN, D. W.; BALDINI, F. D.; MARTIN, P. E.; KOHRT, W. M. Ten kilometer performance and predicted velocity at $\mathrm{VO} 2 \mathrm{max}$ among well-trained male runners. Medicine and Science in Sports and Exercise, Madison, v. 21, n. 1, p. 78-83, 1989.

PASSELERGUE, P. A.; CORMERY, B.; LAC, G.; LEGER, L. A. Utility of the Conconi's heart rate deflection to monitor the intensity of aerobic training. Journal of Strength and Conditioning Research, Philadelphia, v. 20, n. 1, p. 88-94, 2006.

SIMOES, H. G.; GRUBERT CAMPBELL, C. S.; KOKUBUN, E.; DENADAI, B. S.; BALDISSERA, $V$. Blood glucose responses in humans mirror lactate responses for individual anaerobic 
threshold and for lactate minimum in track tests.

European Journal of Applied Physiology and

Occupational Physiology, Basel, v. 80, n. 1, p. 34-40, 1999.

VACHON, J. A.; BASSETT JUNIOR, D. R.;

CLARKE, S. Validity of the heart rate deflection point as a predictor of lactate threshold during running. Journal of Applied Physiology,

Bethesda, v. 87, n. 1, p. 452-459, 1999.

\section{Endereço:}

Fabrizio Caputo

Rua Pascoal Simone, 358, Coqueiros

Florianópolis SC Brasil

88080-350

Telefone: (48) 3321-8641

Fax: (48) 3321-8607

E-mail: fabriziocaputo@hotmail.com

Recebido em: 31 de março de 2010.

Aceito em: 07 de dezembro de 2011.

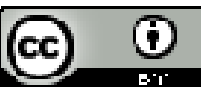

Motriz. Revista de Educação Física. UNESP, Rio Claro,

SP, Brasil - elSSN: 1980-6574 - está licenciada sob Creative Commons - Atribuição 3.0 